\title{
Ação educativa com profissionais de saúde na identificação do idoso vulnerável: relato de experiência
}

\author{
An educational experience in health with health-care professionals to identify vulnerable \\ elderly groups
}

Una experiencia de educación en salud con profesionales de la salud en la identificación del los grupos de adultos mayores

Viviane Ferraz Ferreira de Aguiar ${ }^{1 *}$, Ana Karoline dos Reis Barros ${ }^{1}$, Diego Moraes Barral ${ }^{1}$, Juliane Alves da Silva1 ${ }^{1}$, Tallyssa Taianne da Silva Cardoso ${ }^{1}$, Fernando Conceição de Lima ${ }^{1}$, Thainara Braga Soares ${ }^{1}$ Juliana Raiyanni Sousa Neto ${ }^{1}$.

\section{RESUMO}

Objetivo: Realizar uma ação educativa com profissionais da saúde sobre a utilização do instrumento de identificação do idoso vulnerável, o Índice de Vulnerabilidade Clínico Funcional-20 (IVCF-20). Detalhamento do caso: Trata-se de um estudo descritivo, do tipo relato de experiência, realizado por acadêmicos de enfermagem do 6 período, de uma Instituição Privada de Ensino, na cidade de Belém-Pará, em abril de 2019. A atividade foi realizada com dez profissionais, envolvendo um enfermeiro, quatro Agentes Comunitária da Saúde, uma assistente social e uma psicóloga do Núcleo de Apoio a Saúde da Família (NASF), um médico e duas técnicas de enfermagem. O local da ação foi em uma Unidade de Saúde da Família (ESF), localizado no Distrito Administrativo do Benguí (DABEN), na cidade de Belém-Pará. Utilizaram-se como estratégias para o desenvolvimento da ação educativa a roda de conversa, guiado por materiais ilustrativos como banner e cartilha, e o estudo de um caso clínico. Considerações finais: $O$ resultado da ação educativa evidenciou que o apoio aos profissionais de saúde deve ser realizado visando a melhoria na qualidade do atendimento ao idoso. É necessário que as políticas públicas sejam implementadas para que vise a autonomia e a independência do idoso.

Palavras-Chave: Idoso, Vulnerabilidade em Saúde, Capacitação profissional.

\begin{abstract}
Objective: The aim this study is to report the experience about educational action with health professionals on the use of the instrument of identification of the vulnerable elderly, the Clinical Functional Vulnerability Index-20 (IVCF-20). Case detail: This is a descriptive study, of the type of experience, carried out by nursing students from the 6th period, of a Private Educational Institution, in the city of Belém-Pará, in April 2019. The activity was performed with ten professionals, involving a nurse, four Community Health Agents, a social worker and a psychologist of the Family Health Support Center (NASF), a physician and two nursing techniques. The site of action was in a Family Health Strategy (ESF), located in the Administrative District of Benguí (DABEN), in the city of Belém-Pará. The discussion thread, guided by illustrative materials such as banner and booklet, and the study of a clinical case, were used as strategies for the development of the educational action. Final considerations: The result of the educational action evidenced that the support to health professionals should be carried out aiming at improving the quality of care for the elderly. It is necessary that public policies be implemented so that it aims at the autonomy and independence of the elderly.
\end{abstract}

Keywords: Elderly, Vulnerability in Health, Professional training.

${ }^{1}$ Centro Universitário Metropolitano da Amazônia (UNIFAMAZ), Belém-Pará. *E-mail: viviane.ferraz@yahoo.com.br 


\section{RESUMEN}

Objetivo: Realizar una acción educativa con profesionales de la salud sobre la utilización del instrumento de identificación del anciano vulnerable, el Índice de Vulnerabilidad Clínica Funcional-20 (IVCF-20). En el presente trabajo se analizaron los resultados obtenidos en el análisis de los resultados obtenidos en el análisis de los resultados obtenidos en el estudio. se realizó con diez profesionales, involucrando a una enfermera, cuatro Agentes Comunitarios de la Salud, una asistente social y una psicóloga del Núcleo de Apoyo a la Salud de la Familia (NASF), un médico y dos técnicas de enfermería. El sitio de la acción fue en una Estrategia Salud de la Familia (ESF), ubicado en el Distrito Administrativo del Benguí (DABEN), en la ciudad de BelémPará. Se utilizaron como estrategias para el desarrollo de la acción educativa la rueda de conversación, guiado por materiales ilustrativos como banner y cartilla, y el estudio de un caso clínico. Consideraciones finales: El resultado de la acción educativa evidenció que el apoyo a los profesionales de salud debe ser realizado buscando la mejora en la calidad de la atención al anciano. Es necesario que las políticas públicas sean implementadas para que tenga en cuenta la autonomía y la independencia del anciano.

Palabras clave: Ancianos, Vulnerabilidad en Salud, Capacitación profesional.

\section{INTRODUÇÃO}

A inversão da pirâmide etária tem influenciado no crescimento acelerado do número de pessoas idosas e, consequentemente o aumento significativo da expectativa de vida. Para o ano de 2150 espera-se que o Brasil seja o quinto país com maior população. Diante desta realidade, torna-se necessário o desenvolvimento do envelhecimento ativo (AGUIAR VFF et al., 2019).

Contudo, verifica-se que há uma falta de cuidado especializado voltado ao idoso, que pode ser agravada pela presença de doenças crônicas não transmissíveis, ocasionando baixa qualidade de vida e incapacidade funcional, tornando o idoso vulnerável. (BINOTTO MA et al., 2018), (MELO EMA et al., 2018).

A vulnerabilidade está relacionada as limitações que o idoso pode apresentar ocasionando declínios relacionados ao âmbito biológico, socioeconômico, psicossocial e cultural. A vulnerabilidade do idoso o leva à fragilidade estando mais propenso a desenvolver complicações. (BARBOSA KTF, 2017), (TAVARES RE et al., 2017).

Para Moraes EN (2016), a identificação precoce da vulnerabilidade nos idosos pode se dar pelo reconhecimento das alterações do envelhecimento que compromete a capacidade de homeostase do idoso quando submetido a situações de estresse. Sendo assim, o reconhecimento das causas e motivos para a ocorrência do estado vulnerável permite o planejamento e implementação de ações e serviços de saúde que atendam de forma resolutiva as demandas da população. (MAIA FOM et al., 2012).

A Política Nacional de Saúde da Pessoa Idosa (PNSPI) destaca em suas diretrizes a necessidade de se manter a autonomia e independência do idoso, portanto, deve ser realizada avaliação multidimensional deste público-alvo, visando a identificação das alterações do envelhecimento que estejam associadas a situações de fragilidade (MARQUES GCS et al., 2018).

Neste contexto, torna-se necessário a capacitação e formação dos profissionais de saúde na área de saúde da pessoa idosa. Todavia, de acordo com Paiva EP et al. (2016) os profissionais ainda não possuem formação específica para o atendimento a pessoa idosa, conforme identificado em sua pesquisa.

É válido ressaltar que é imprescindível a aquisição de estratégias para auxiliar o profissional na consulta e os instrumentos de avaliação multidimensional, com sua aplicação simples, rápida e clara contribuem para a eficiência no diagnóstico e intervenção precoce em problemas de saúde que oferecem risco a qualidade de vida e autonomia dos idosos (BISPO EPF et al., 2012). Entre estes instrumentos está o Índice de Vulnerabilidade Clínico Funcional-20 (IVCF-20), utilizado para auxiliar o profissional na identificação precoce da vulnerabilidade. É o primeiro instrumento desenvolvido no Brasil por profissionais de saúde que tem como 
finalidade de realizar a identificação do idoso frágil, pois avalia a idade, autopercepção da saúde, atividades de vida diária (Atividade Básica de Vida Diária e Atividade Instrumental de Vida Diária), cognição, humor, mobilidade, comunicação e comorbidades múltiplas (MORAES EN et al., 2016).

Neste contexto, o estudo justifica-se por haver a necessidade de se implementar estratégias que auxiliem a identificação do idoso vulnerável de forma rápida, simples e eficaz. Portanto, o objetivo deste estudo foi realizar uma ação educativa com profissionais da saúde sobre a utilização do instrumento de identificação do idoso vulnerável, o Índice de Vulnerabilidade Clínico Funcional-20 (IVCF-20).

\section{DETALHAMENTO DO CASO}

Trata-se de um estudo descritivo, do tipo relato de experiência, realizado por acadêmicos de enfermagem do 6 o período, de uma Instituição Privada de Ensino, na cidade de Belém-Pará, durante abril de 2019. A temática escolhida surgiu a partir das aulas de Enfermagem na Atenção à Saúde do Idoso e discussão sobre os instrumentos de avaliação geriátrica. Buscou-se o levantamento de artigos científicos nas bases de dados Literatura Latino-Americana e do Caribe em Ciências da Saúde (Lilacs) e Scientific Electronic Library Online (Scielo), manuais do Ministério da Saúde sobre o idoso e a leitura do Índice de Vulnerabilidade Clínico Funcional-20 (IVCF-20). Após o levantamento dos materiais, a questão norteadora levantada foi: Qual a importância da capacitação para utilizar o instrumento Índice de Vulnerabilidade Clínico Funcional-20 (IVCF20) na identificação do idoso vulnerável?

A atividade foi realizada com dez profissionais, envolvendo um enfermeiro, quatro Agentes Comunitária da Saúde, uma assistente social e uma psicóloga do Núcleo de Apoio a Saúde da Família (NASF), um médico e duas técnicas de enfermagem. O local da ação foi em uma Estratégia Saúde da Família (ESF), localizado no Distrito Administrativo do Benguí (DABEN), na cidade de Belém-Pará. Utilizaram-se como estratégias para o desenvolvimento da ação educativa a roda de conversa e o estudo de um caso clínico. O primeiro momento foi guiado por materiais ilustrativos como banner, contendo a ficha-espelho do instrumento de Identificação do doso vulnerável IVCF-20 e a apresentação de uma cartilha ilustrativa, construídos pelos pesquisadores. A cartilha teve como intuito facilitar o desenvolvimento da ação, contendo alguns questionamentos como: "o que é IVCF-20?", "onde e como foi criado?", "quais profissionais podem utilizar"?" e "como aplicá-lo?".

Devido a importância da utilização de tecnologias que sejam facilitadoras do atendimento ao usuário do serviço de saúde, utilizou-se o celular dos participantes para a instalação do aplicativo IVCF-20. Este momento teve como intuito mostrar que o instrumento não precisa estar impresso, sendo assim facilita a sua utilização em qualquer momento da consulta tanto na ESF como na visita domiciliar.

Após o compartilhamento de conhecimentos e experiências foi discutido sobre um caso clínico em que os profissionais deveriam levantar os problemas identificados na idosa do caso e os itens a serem avaliados no IVCF-20. A descrição do caso clínico envolvia uma idosa, 88 anos, que relatava saúde muito boa, mas que a acompanhante informou ao contrário, enfatizando que a saúde da idosa era regular e que deixou de fazer algumas atividades diárias como cozinhar e limpar sua casa. No caso clínico também foi descrito que a idosa costumava participar de grupos de apoio, duas vezes por semana, onde realizava trabalho de crochê. Faz uso de duas medicações para hipertensão arterial, relata dormir bem e tem uma alimentação saudável.

A utilização dos dois momentos, roda de conversa e caso clínico foi importante para evidenciar a importância de se conhecer formas de avaliação quanto a vulnerabilidade do idoso. Verificou-se que os profissionais desconheciam sobre o instrumento de avaliação da vulnerabilidade do idoso. Mas que perceberam a sua importância no atendimento devido a avaliação criteriosa que o instrumento permite realizar. A estratégia utilizada pelos pesquisadores facilitou a socialização do conhecimento e uma resposta significativa. Durante a capacitação profissional acerca do instrumento índice de avaliação clínico funcional 20 (IVCF-20) percebemos ser uma ferramenta útil e eficaz de importância significativa para o rastreamento rápido do idoso frágil. Considera-se que esta experiência foi uma ação motivacional para que as atividades de capacitação sejam realizadas para os profissionais, pois o aumento da população idosa é uma vertente e que precisa ser discutida.

REAS/EJCH |Vol. Sup. 28 | e932 | DOI: https://doi.org/10.25248/reas.e932.2019 Página 3 de 5 


\section{DISCUSSÃO}

O IVCF 20 é um instrumento de avaliação do idoso frágil, sua avaliação envolve aspectos relacionados a faixa etária do idoso, autopercepção da saúde, identificação das atividades de vida diária, alterações de cognição e humor, mobilidade, comunicação e comorbidades. Identifica-se que é um questionário amplo, por esta razão é conhecido como multidimensional. Sua pontuação total é igual a 40 , sendo este valor considerado o risco máximo que o idoso pode apresentar quanto sua vulnerabilidade clínico-funcional do idoso. É válido ressaltar que, apesar de ser considerado um instrumento de rastreio, é necessário que o idoso seja acompanhado por uma equipe especializada na área da geriatria e gerontologia (MORAES EN et al., 2016).

O aumento da vulnerabilidade influencia na qualidade de vida do idoso, pois advém de uma combinação de fatores que culminam na elevada suscetibilidade ao adoecimento e morte. (BARBOSA KTF et al., 2017). Nota- se que sua utilização ainda é insuficiente e que os profissionais da saúde precisam se empoderar deste instrumento. Em uma pesquisa realizada por Meireles VC e Baldissera VDA (2019) sobre a qualidade dos cuidados aos idosos frágeis com risco para lesão por pressão em uma atenção primária à saúde verificou-se que os profissionais de saúde não utilizam o IVCF-20 tendo como justificativa a redução do número de profissionais, contudo, para os pesquisadores a não efetivação de uma avaliação multidimensional promove um cuidado fragmentado, além do que a falta de estratificação do risco diminui a possibilidade de encaminhamento deste idosos para serviços especializados.

Tendo em vista a importância do IVCF- 20 na busca de confidencialidade e validade, a sua compreensão promove a integração e investigação de possíveis fragilidades no idoso. Sabe-se que profissionais com competência da informação estão melhor preparados para utilizá-lo com eficiência e eficácia, visto que que este instrumento pode ser utilizado por qualquer profissional da área da saúde, devidamente capacitado. Desta maneira, considera-se uma contribuição significativa para a manutenção da saúde da pessoa idosa. (PEREIRA AMVB; ROSA ACDS 2018).

\section{CONSIDERAÇÕES FINAIS}

O resultado da ação educativa evidenciou que o apoio aos profissionais de saúde deve ser realizado visando a melhoria na qualidade do atendimento ao idoso. É necessário que as políticas públicas sejam implementadas para a promoção da autonomia e independência do idoso. A experiência dos pesquisadores quanto compartilhar o conhecimento com os participantes e evidenciar que há instrumentos que podem facilitar o processo de trabalho foi fundamental para o crescimento acadêmico e valorização dos instrumentos de avaliação geriátrica para a condução das consultas.

\section{REFERÊNCIAS}

1. AGUIAR VFF, et al. Intergenerationally between the elderly and adolescents in the search for the deconstruction of stereotypes in old age: experience report. Revista Eletrônica Acervo Saúde, 2019; (23), e413.

2. BARBOSA KTF et al. Envelhecimento e vulnerabilidade individual: um panorama dos idosos vinculados à estratégia saúde da família. Texto Contexto Enferm, 2017; 26 (2):1-10, e2700015.

3. BINOTTO MA, et al. Physical frailty and gait speed in community elderly: a systematic review. Rev Esc Enferm USP. 2018;52: e03392.

4. BISPO EPF. Avaliação da capacidade funcional de idosos cadastrados na Estratégia e Saúde da Família na comunidade do Pontal da Barra, Maceió - AL. Cad. Ter. Ocup. UFSCar, São Carlos, 2012; 20(1): 81-87.

5. MAIA FOM, et al. Adaptação transcultural do Vulnerable Elders Survey -13 (VES-13): contribuindo para a identificação de idosos vulneráveis, Rev Esc Enferm USP, 2012; 46(Esp):116-22.

6. MARQUES GCS, et al. Profissional Enfermeiro: Competências e habilidades para a avaliação multidimensional da pessoa idosa. Revista Kairós-Gerontologia, 2018; 21(2): 307-326.

7. MEIRELES VC, BALDISSERA VDA. Qualidade da atenção aos idosos: risco de lesão por pressão como condição marcadora. Rev Rene. 2019;20: e40122. 
8. MELO EMA, et al. Frailty syndrome and associated factors in elderly residents in long-term institutions. Saúde Debate, 2018; (42)117: 468-480.

9. MORAES EN et al. Índice de Vulnerabilidade Clínico Funcional-20 (IVCF-20): reconhecimento rápido do idoso frágil. Rev Saúde Pública, 2016;50:81.

10. PAIVA EP. Assistência dos enfermeiros ao idoso: um estudo transversal. HU Revista, Juiz de Fora, 2017; 42(4):259265.

11. PEREIRA AMVB, ROSA ACDS. Linha guia da saúde do idoso / SAS-SESA. Curitiba: SESA, 2018.

12. RIBEIRO EG, et al. Self-perceived health and clinical functional vulnerability of the elderly in Belo Horizonte/Minas Gerais. Rev Bras Enferm [Internet], 2018;71(suppl 2):860-7.

13. TAVARES RE et al. Ações de enfermagem ao idoso na estratégia saúde da família: revisão integrativa. Rev de Enferm [Internet],2018; (11) 2:1052-61. 